\title{
In-situ TEM Observation of Synthesizing Long Gold Atom Strand
}

\author{
Y. Oshima*, Y. Kurui**, H. D. Nguyen***, T. Ono*** and K. Takayanagi** \\ * Research center for ultra HVEM, Osaka University, 7-1 Midorigaoka, Ibaraki, 567-0047, Japan \\ ** Department of Condensed Matter Physics, Tokyo Institute of Technology, 2-12-1-H-51 Oh- \\ okayama, Meguro-ku, Tokyo 152-8551, Japan \\ *** Department of Precision Science and Technology, Osaka University, Suita, Osaka 565-0871, Japan.
}

Metal atom strands show unique physical and chemical properties because of one-dimensional material. They have been typically synthesized by pulling a thin metal neck suspended between two electrodes, as demonstrated in mechanical controllable breaking junction (MCBJ) experiments. During pulling, the metal neck is discontinuously thinned due to plastic deformation until it breaks. A pure gold atom strand has been observed to be a maximum of 2 or 3 atoms in length [1]. On the other hand, theoretically, the Au-Au-O-Au-Au bond has been suggested to be so strong that more gold atoms can be extracted from the electrode upon pulling [2,3]. Other than the oxygen atom, it has been suggested that hydrogen and carbon atoms could be incorporated to form long gold atom strands. Experimentally, long gold atom strands was reported to be synthesized by extracting gold atoms one by one from an electrode surface [4], which was suggested to incorporate either oxygen atoms or hydrogen molecules. But, it is difficult to experimentally detect the incorporation of such impurity atoms in the strands, since these are light elements.

In this study, using an ultra high-vacuum transmission electron microscope (UHV-TEM) combined with a scanning tunneling microscope (STM) [5], we observed the synthesis of long gold atom strands from a carbon-contaminated gold surface, as shown in Fig.1, at room temperature. Since the UHV condition prevented the presence of residual gases, such as oxygen, nitrogen and hydrogen molecules, in the microscope, the observed long gold strand was formed by the incorporation of carbon atoms. First-principles calculations showed that the long strand has two carbon atoms at each gold atom interval.

The carbon atoms on the carbon-contaminated gold surface were observed to clump during observation, indicating that carbon atoms do not have a strong bond with the gold atoms. When sandwiched between two electrodes along with application of a relatively high bias voltage (0.5-1 $\mathrm{V}$ ), agglomerates of carbon atoms change into graphitized structures, like graphene, nanotubes and fullerenes, due to Joule heating, as reported previously [6]. The carbon fullerene shown in Fig. 1 was formed by Joule heating. A long gold atom strand was then synthesized, when pulling out gold atoms from a carbon-contaminated surface with the application of a relatively low bias voltage (13 $\mathrm{mV}$ ). However, the gold atom strand was only synthesized when the amount of carbon atoms on the surface was approximately lower than one monolayer coverage. When the amount of carbon atoms was large and the bias voltage was low, the carbon atoms just clumped together at a certain point. In Fig. 1, after reducing the amount of carbon atoms on the surface by forming the fullerene, the long strand could be synthesized.

Figure 1 shows a typical TEM series of the synthesis of long gold atom strand. The dark dots in the TEM images were identified to be gold atoms. In the simulated TEM image of Fig. 1(f), a dark contrast corresponding to a gold atom has a similar intensity to the fullerene attached to the upper electrode. Since the dark dots aligned regularly in the strand has also similar contrast as the fullerene in the observation, they were identified as being gold atoms. The number of gold atoms in the strand increases one by one from 3 to 7 (Fig. 1(a)-(e)). The piezo-displacement for introducing each gold 
atom was estimated to be $0.5 \mathrm{~nm}$, equal to the atom interval estimated in the image. Such a long interval has been already reported in the previous TEM observation [7]. The observed strand in the present study was confirmed by first-principles calculation to have two carbon atoms in each $0.5 \mathrm{~nm}$ interval between two gold atoms. In the case of the freestanding atom strand, when the number of carbon atoms incorporated between gold atoms was one, two, and three, the distance between two gold atoms was calculated to be $0.4,0.5$, and $0.64 \mathrm{~nm}$, respectively.

Another TEM series (not shown) of the synthesis of a long atom strand showed that the gold (111) surface layer of the electrode was modified due to the incorporation of carbon atoms and it was gradually removed from the bottom electrode in the pulling process. This result suggested that the long strand may be constructed along the [112] direction on the carbon-adsorbed gold (111) surface as a stable one-dimensional configuration before pulling. We calculated the formation energy of a gold strand incorporating one, two or three carbon atoms at each gold atom interval on gold (111) surface and found that a gold strand incorporating two carbon atoms at each gold atom interval $(0.5 \mathrm{~nm})$ was lower in the formation energy. This strand is constructed along the [112] direction on the carbon-adsorbed gold (111) surface before pulling. These results suggest a new paradigm for synthesizing strong atom strands.

\section{References}

[1] U. Landman, et al., Science 248 (1990) 454.

[2] F. D. Novaes, et al., Phys. Rev. Lett. 96 (2006) 016104.

[3] W. H. Thijssen, et al., Phys. Rev. Lett. 96 (2006) 026806.

[4] T. Kizuka, Phys. Rev. B 77 (2008) 155401.

[5] Y. Oshima, et al., Surf. Sci. 531 (2003) 209.

[6] M. Yoshida, M. et al., Jpn J. Appl. Phys. 46 (2007) L67.

[7] S. B. Legoas, et al., Phys. Rev. Lett. 88 (2002) 076105.

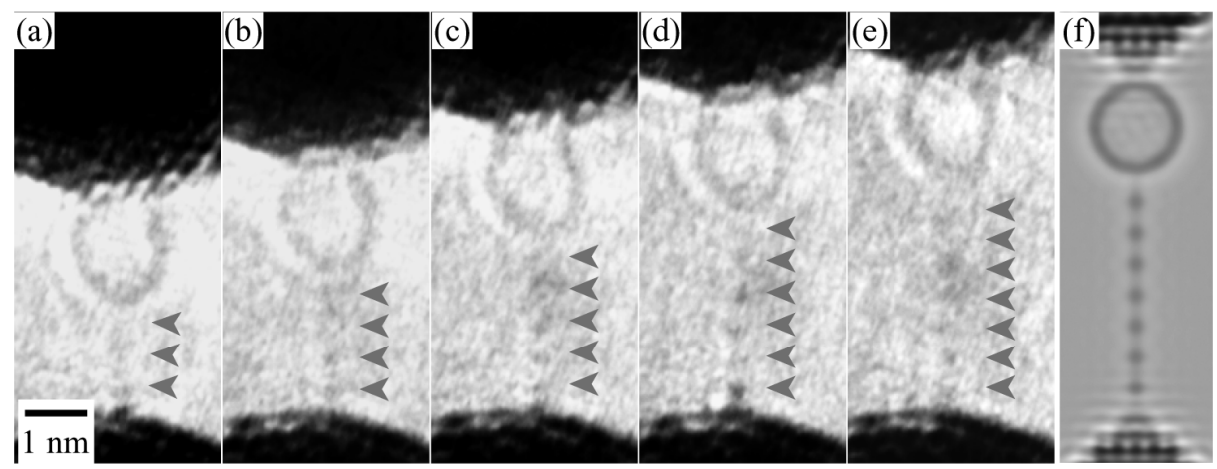

Fig. 1 (a) - (e) A series of TEM images taken at each introduction of a gold atom to the strand during the pulling the gold atoms from the bottom electrode. The number of gold atoms, indicated by arrows, increases from 3 to 7 . The distance between two gold atoms is $0.5 \mathrm{~nm}$. (f) Simulated TEM image of the strand consisting of 7 gold atoms. The fullerene is assumed to be $\mathrm{C}_{240}$. 